\title{
BioMedicine
}

Volume 10 | Issue 3

Article 2

2020

\section{A Review on Recent Advancements in Diagnosis and Classification of Cancers Using Artificial Intelligence}

Follow this and additional works at: https://www.biomedicinej.com/biomedicine

Part of the Life Sciences Commons, and the Medicine and Health Sciences Commons (c) (i)

This work is licensed under a Creative Commons Attribution 4.0 License.

\section{Recommended Citation}

Ramesh, Priyanka; Karuppasamy, Ramanathan; and Veerappapillai, Shanthi (2020) "A Review on Recent Advancements in Diagnosis and Classification of Cancers Using Artificial Intelligence," BioMedicine: Vol. 10 : Iss. 3 , Article 2.

DOI: $10.37796 / 2211-8039.1012$

This Review Articles is brought to you for free and open access by BioMedicine. It has been accepted for inclusion in BioMedicine by an authorized editor of BioMedicine. 
A Review on Recent Advancements in Diagnosis and Classification of Cancers Using Artificial Intelligence

\section{Cover Page Footnote}

The authors thank management of Vellore Institute of Technology, Vellore for providing the facilities to carry out this work. 


\title{
A review on recent advancements in diagnosis and classification of cancers using artificial intelligence
}

\author{
Priyanka Ramesh, Ramanathan Karuppasamy, Shanthi Veerappapillai*
}

Department of Biotechnology, School of Bio Sciences and Technology, Vellore Institute of Technology, Vellore, 632 014, Tamil Nadu, India

\begin{abstract}
Artificial intelligence has illustrated drastic changes in radiology and medical imaging techniques which in turn led to tremendous changes in screening patterns. In particular, advancements in these techniques led to the development of computer aided detection (CAD) strategy. These approaches provided highly accurate diagnostic reports which served as a "second-opinion" to the radiologists. However, with significant advancements in artificial intelligence strategy, the diagnostic and classifying capabilities of CAD system are meeting the levels of radiologists and clinicians. Thus, it shifts the CAD system from second opinion approach to a high utility tool. This article reviews the strategies and algorithms developed using artificial intelligence for the foremost cancer diagnosis and classification which overcomes the challenges in the traditional method. In addition, the possible direction of AI in medical aspects is also discussed in this study.
\end{abstract}

Keywords: Artificial intelligence, Artificial neural network, Breast cancer, Gastric cancer, Lung cancer

\section{Introduction}

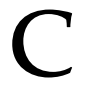
ancer being one of the non-communicable diseases is ranked at the foremost for being the blockade of survival rate among the global population. For instance 1.8 million new cases and 9.6 million deaths were estimated worldwide in 2018. Among them $59.5 \%$ of global cancer deaths had occurred in Asia. Moreover cancer in lung, breast, pancreatic, gastric and skin were the significant contributors for the highest incidence rate in 2018 [1]. Due to the rise of cancer incidence rate, diagnosing of disease using conventional tools at early stage had become difficult. Moreover, these traditional methods experienced diagnostic errors including missed, wrong and delayed cases [2]. On the other hand, understanding the perplexity of cancer at different stages has complicated the research further. The perplexity of cancer includes early detection, accuracy, tumor evolution, metastasis pattern, recurrence, tumor aggressiveness and determination of tumor margins [3]. To overcome the limitations mentioned above and to diagnose cancer at the earliest, advancement in artificial intelligence (AI) had been raised for quantifying the imaging data.

Deep learning, a section in AI, plays a promising role in automated recognition of features from sample medical images beyond human's role in particular tasks [4]. For instance automated applications of $\mathrm{AI}$ enhanced the qualitative potentials of clinicians, which includes tracing numerous lesions at a time, prediction of the resultant tumor by referring to the various databases within a short period, translation of phenotypic variations to genotypic and persistent monitoring of patients. Despite the need for a large quantity of data for training, deep learning had illustrated relative

Received 11 April 2020; accepted 16 June 2020.

Available online 11 September 2020.

* Corresponding author at. Fax: +0416 2243092

E-mail address: shanthi.v@vit.ac.in (S. Veerappapillai). 


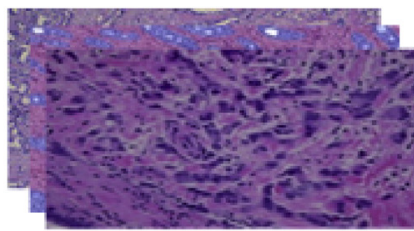

Digitally Scanned Cancer Images

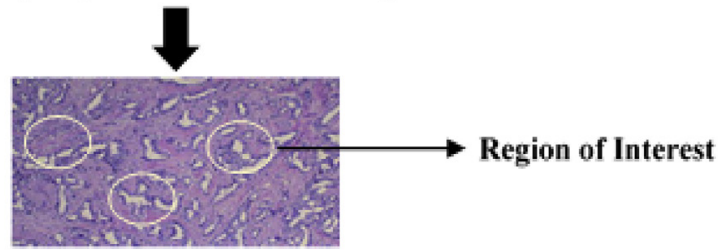

Image Preprocessing $\longrightarrow$ Image enhancement

- Region extraction

- Feature extraction

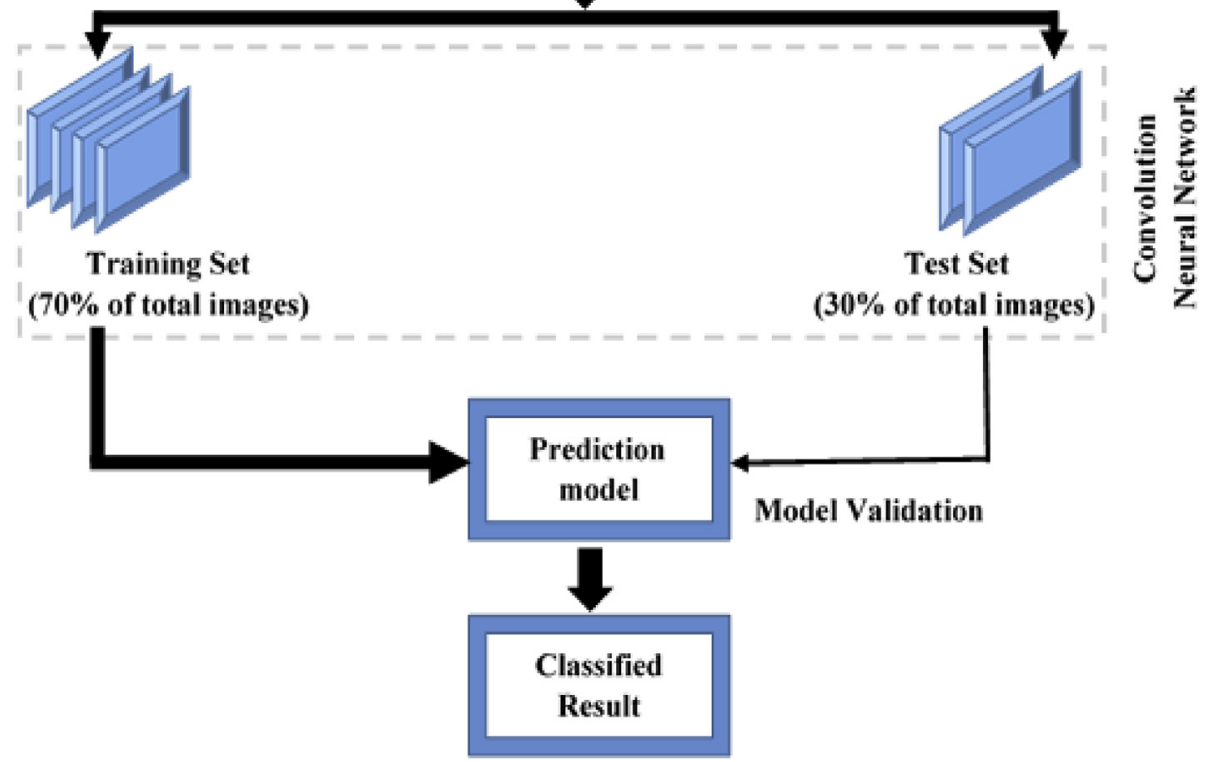

Fig. 1. Overall process involved in tumor diagnosis and classification.

stability against random fluctuations in the ground truth features [5]. Furthermore it generalizes disease imaging techniques with minimal errors which in turn lead to earlier and significant diagnosis of diseases.

Besides deep learning, artificial neural network (ANN) is also used to classify cancer more precisely. It is a mathematical model postulated from the human nervous system comprising of interconnected neurons. Connectionist strategy is implemented in this technique for computational processing of information. Eventually to illustrate the adaptive system, neural network plays a significant role by modifying its structure based on the training data and by detecting patterns in the data. ANN can be designed through learning process for any particular application [6]. On learning the data during its training time, it organizes itself to proceed further. Moreover many networks can be performed simultaneously and also bears fault tolerance by preventing degradation of structures [7]. Though these studies are intensively applicable in pre-clinical studies, the automated approach of tumor diagnosis and classification discriminates the cancer period of time. The overall process involved in tumor diagnosis and classification were briefed in Fig. 1.

At the light of these evidences, current studies and future applications of AI to medical imaging in top incidental cancers were reviewed during this investigation. This paper highlights the application of AI that generated a drastic transformation in 
Theoretical framework

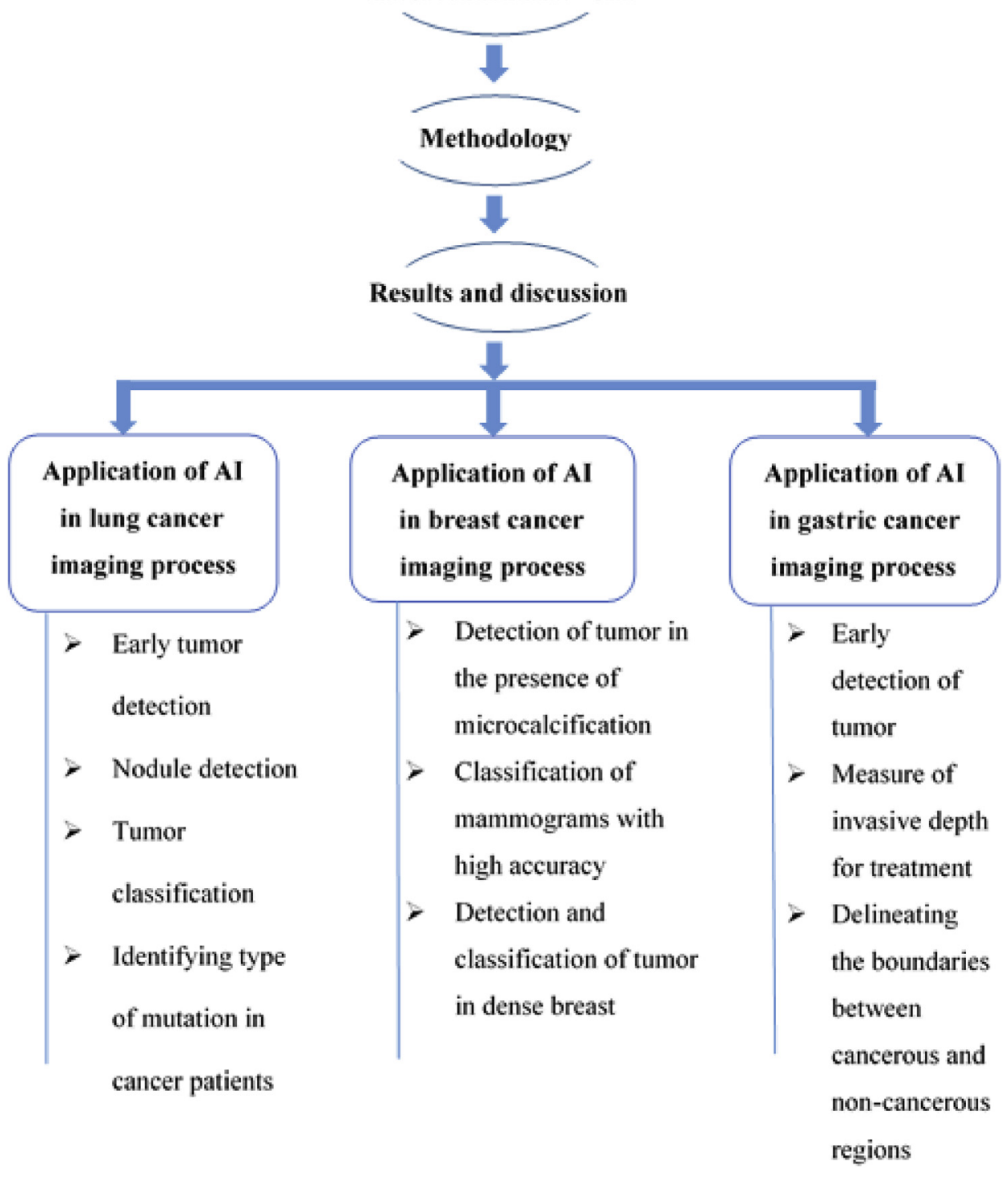

Fig. 2. Schematic representation illustrating overall view of our study.

cancer diagnosis and treatment. In particular, the study includes case studies which enlightens application of AI in diagnosis and classification of three cancers (lung, breast and gastric cancer). The evolution of $\mathrm{AI}$ in cancer imaging described in this study also provides the significance of AI in cancer diagnosis and treatment. Moreover this study also provides potential applications of AI overcoming the limitations in cancer imaging including presence of denser tissues during diagnosis and classification of cancerous samples from non-tumor samples. The number of case studies concerning AI technology in lung, breast and gastric cancer clarifies the other characteristics of this latest technology. Overall, the study shows the significant application of AI technology which in turn improves the cancer care in the society. Moreover, this paper also suggests that artificial intelligence application in cancer imaging 
needs further evaluation and validation for improved reliability and generalizability of approaches in clinical practices [8]. The tree diagram provided in Fig. 2 illustrates the structure of the paper for better understanding.

\section{Theoretical background}

AI in oncology imaging process includes three major clinical tasks: Diagnosis, characterization and monitoring. In general, detection involves observation of pathologists over variations in image intensities or complex patterns to understand the aberrations in the patients. Advanced computational technology-assisted pathologists to predict and identify cancer with more accuracy and fewer errors. This strategy of detection is known as Computer Aided Detection (CAD) [9]. AI based tools assisted in identification of missed cancer patients as well as detection with high accuracy and sensitivity [10]. The second task characterization includes diagnosis, segmentation and staging of cancer. This task quantifies abnormality of features including size and texture. Segmentation measures extent of abnormalities in a two dimensional (2D) plane. Manual segmentation process in clinical practice limits itself with minimal accuracy, interrater bias, high time and labor consumption and inconsistent reproducibility [11]. In contrast, AI based approach provided automated segmentation with increased quality, reproducibility and efficiency.

Further expansion of technology leads to an integrated method in which separate segmentation process is not required. This task also includes staging of disease in which the cancer is explained based on predefined features and are classified based on TNM classification [12]. Ensemble methods were employed in traditional staging task whereas automated staging relies on tumor size, metastasis and neighboring lymph node data for classification [13].

On the other hand ANN had become a significant strategy for cancer classification. It consists of three consecutive layers namely input layers, hidden layers and output layer. ANN can be classified into single layer feed forward network, single node with its own feedback, multi-layer feed forward network and multi-layer recurrent network. In addition, it also uses three different learning strategy for generating neural network which includes supervised learning, unsupervised learning and reinforcement learning. Among them supervised learning has higher accuracy and precision as it develops output based on the pattern of each input
[7]. Hence automated staging is more reliable than traditional approach. Despite the complexity of data, artificial intelligence is remarkable in identifying the features from large volume of medical data which in turn is used to assist clinicians [14]. It also identifies complicated patterns and supports in transforming images into valuable quantitative information. It is to be noted that these information are not recognized by humans, thus makes clinical decisions easier.

Besides, detection and classification of cancers were the major challenges in the medical field. Artificial intelligence assists clinicians in interpreting cancer images, including cancer stages, tumor delineations, detection of mutations, the impact of anti-cancer treatment, and the influence of disease on other organs. For instance, Khan et al. had proposed an integrated convolution neural network algorithm to classify the breast tumor as benign and malignant, which in turn reduced the burden of pathologists in tumor classification [15]. Similarly, a deep learning model developed by Kim et al. and his co-workers assisted the clinicians in predicting the survival rate of the oral cancer patients, which in turn helped the medical practitioners to provide appropriate and effective treatments to the patients [16].

Moreover, medical input data varies beyond imaging techniques, which include blood biomarkers, molecular signatures, and statistical data. It is to be noted that AI is one of the integrative tools that parallel and normalizes various streams of information. Also, advancements in AI approach assists human experts to visualize, understand, and analyze the results [17]. Besides medical imaging, the application of $\mathrm{AI}$ in health monitoring enhances efficiency with reduced cost.

Despite radiation dose, subsequent measurements, and scan - time, advancements in phasecontrast imaging techniques can assists radiologists

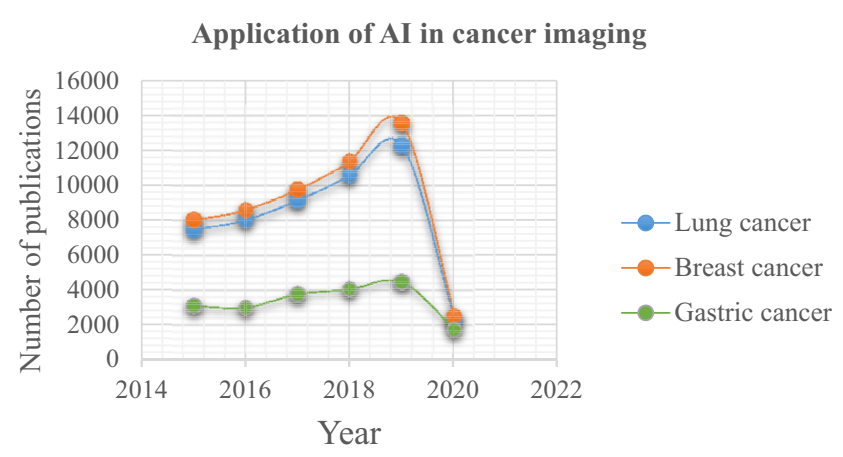

Fig. 3. Graph explaining the significance of AI in the field of cancer imaging. 
Table 1. Recently proposed algorithms for early diagnosis and classification of lung cancer.

\begin{tabular}{|c|c|c|c|c|c|c|c|}
\hline S. No & Paper & Year & Input images & Dataset & Purpose & Classifier & Results \\
\hline 1 & ALzubi et al. [28] & 2019 & $\begin{array}{l}\text { Thoracic surgery } \\
\text { dataset }\end{array}$ & 1200 & Lung cancer diagnosis & $\begin{array}{l}\text { Weight Optimized NN with } \\
\text { Maximum Likelihood } \\
\text { Boosting classification }\end{array}$ & Feature selection rate $-90 \%$ \\
\hline 2 & Pandiangan et al. [29] & 2019 & $\mathrm{X}$-ray images & 40 & Lung cancer detection & ANN & Accuracy - 99\% \\
\hline 3 & Nasser et al. [30] & 2019 & Lung cancer dataset & NA & Lung cancer detection & $\begin{array}{l}\text { Feed forward back } \\
\text { propagation neural network }\end{array}$ & Accuracy $-96.67 \%$ \\
\hline 4 & Roy et al. [31] & 2019 & Lung CT images & 100 & Lung cancer detection & $\begin{array}{l}\text { SVM and Random forest } \\
\text { algorithm }\end{array}$ & $\begin{array}{l}\text { Efficacy }-94.5 \% \\
\text { Sensitivity }-74.2 \% \\
\text { Specificity }-77.6 \%\end{array}$ \\
\hline 5 & Bhalerao et al. [32] & 2019 & Lung CT images & 90 & Lung cancer detection & $\begin{array}{l}\text { Maxpooling and ReLU } \\
\text { algorithm }\end{array}$ & $\begin{array}{l}\text { Accuracy }-94.34 \% \\
\text { Sensitivity }-91.755 \\
\text { Specificity }-95.7 \% \\
\text { Precision }-91.75 \%\end{array}$ \\
\hline 6 & Senthil et al. [26] & 2018 & Lung cancer image dataset & NA & $\begin{array}{l}\text { Early detection of lung } \\
\text { cancer }\end{array}$ & Partial swarm optimization & $\begin{array}{l}\text { Accuracy - } 97.8 \% \\
\text { Sensitivity }-94.8 \%\end{array}$ \\
\hline 7 & Perumal et al. [33] & 2018 & Lung $\mathrm{CT}$ images & 100 & $\begin{array}{l}\text { Lung cancer detection and } \\
\text { classification }\end{array}$ & $\begin{array}{l}\text { Artificial bee colony } \\
\text { optimization }\end{array}$ & $\begin{array}{l}\text { Sensitivity - } 92 \% \\
\text { True positivity rate }-92 \% \\
\text { False error rate }-7.6 \%\end{array}$ \\
\hline 8 & Xin Li et al. [34] & 2018 & Chest CT images & NA & Stage 1 diagnosis & $\mathrm{CNN}$ & $\begin{array}{l}\text { Sensitivity }-96.4 \% \\
\text { Specificity }-95.6 \%\end{array}$ \\
\hline 9 & Wang et al. [25] & 2018 & Histopathology images & 539 & $\begin{array}{l}\text { Discovery of tumor shape and } \\
\text { boundary }\end{array}$ & $\mathrm{CNN}$ & Accuracy $-89.8 \%$ \\
\hline 10 & Coudray et al. [27] & 2017 & Histopathology images & 1175 & $\begin{array}{l}\text { Classification and mutation } \\
\text { predication in NSCLC }\end{array}$ & Inception v3 & $\begin{array}{l}\text { Sensitivity - } 97 \% \\
\text { Specificity - } 97 \%\end{array}$ \\
\hline
\end{tabular}

NN - Neural Network, ANN - Artificial Neural Network, SVM - Support Vector Machine, CNN - Convolution Neural Network, NSCLC - Non Small Cell Lung Cancer. 
Table 2. Recently proposed algorithms for early diagnosis and classification of breast cancer.

\begin{tabular}{|c|c|c|c|c|c|c|c|}
\hline S. No & Paper & Year & Input Data type & Dataset & Purpose & classifier & Results \\
\hline$\overline{1}$ & Batra et al. [48] & 2020 & Mammograms & 161 & Breast cancer detection & Max pooling & $\begin{array}{l}\text { Accuracy (Tensorflow) - } \\
87.98 \% \\
\text { Accuracy (Matlab) - } \\
84.02 \%\end{array}$ \\
\hline 2 & Ali et al. [49] & 2020 & Mammograms & 50 & Breast cancer classification & $\begin{array}{l}\text { Tetrolet transform based k- } \\
\text { means classifier }\end{array}$ & $\begin{array}{l}\text { Accuracy }-92 \% \\
\text { Sensitivity - } 88 \% \\
\text { Specificity - } 96 \%\end{array}$ \\
\hline 3 & Kim et al. [50] & 2020 & Mammograms & 17230 & Detection of breast cancer & $\mathrm{CNN}$ & Accuracy $-95.9 \%$ \\
\hline 4 & Wadkar et al. [51] & 2019 & Mammograms & 5000 & Breast cancer detection & ANN and SVM & $\begin{array}{l}\text { Accuracy (artificial neural } \\
\text { network) - } 97 \% \\
\text { Accuracy (Support Vector } \\
\text { Machine) - } 91 \%\end{array}$ \\
\hline 5 & Alejandro et al. [52] & 2019 & Mammograms & 240 & $\begin{array}{l}\text { Detection and classification } \\
\text { of breast cancer }\end{array}$ & $\mathrm{CNN}$ & $\begin{array}{l}\text { Accuracy - } 89 \% \\
\text { Sensitivity }-86 \% \\
\text { Specificity }-79 \%\end{array}$ \\
\hline 6 & Alickovic et al. [53] & 2019 & Breast cancer dataset & 699 & $\begin{array}{l}\text { Detection and classification } \\
\text { of breast cancer }\end{array}$ & Perceptron neural network & Accuracy $-99.27 \%$ \\
\hline 7 & Rodriguez-Ruiz et al. [54] & 2019 & $\begin{array}{l}\text { Mammograms and breast } \\
\text { tomosynthesis }\end{array}$ & 9000 & $\begin{array}{l}\text { Detection of calcifications } \\
\text { and soft lesions }\end{array}$ & Features classifier & Accuracy $-84 \%$ \\
\hline 8 & Watanabe et al. [55] & 2019 & Breast cancer dataset & 317 & Breast cancer detection & $\begin{array}{l}\text { Artificial intelligence-based } \\
\text { computer-aided detection }\end{array}$ & Accuracy - $90 \%$ \\
\hline 9 & Wang et al. [56] & 2019 & Mammograms & 400 & Breast cancer detection & $\begin{array}{l}\text { Unsupervised extreme } \\
\text { learning machine classifier }\end{array}$ & $\begin{array}{l}\text { Accuracy of Single feature } \\
\text { model - } 76.25 \% \\
\text { Double feature model - } \\
80.75 \% \\
\text { Multi feature model - } \\
84.5 \%\end{array}$ \\
\hline 10 & Huang et al. [57] & 2017 & Breast cancer dataset & 102993 & Breast cancer prediction & SVM & Accuracy - $99.41 \%$ \\
\hline
\end{tabular}

CNN - Convolution Neural Network, ANN - Artificial Neural Network, SVM - Support Vector Machine. 
Table 3. Recently proposed algorithms for early diagnosis and classification of gastric cancer.

\begin{tabular}{|c|c|c|c|c|c|c|c|}
\hline S. No & Paper & Year & Input data type & Dataset & Purpose & Classifier & Results \\
\hline 1 & Aslam et al. [69] & 2020 & Saliva & 220 & $\begin{array}{l}\text { Classification of gastric } \\
\text { cancer into early and } \\
\text { advanced stage }\end{array}$ & SVM & $\begin{array}{l}\text { Accuracy - } 97.18 \% \\
\text { Sensitivity }-96.88 \% \\
\text { Specificity }-97.44 \%\end{array}$ \\
\hline 2 & Li et al. [70] & 2019 & Endoscopic images & 2429 & $\begin{array}{l}\text { Early diagnosis of gastric } \\
\text { cancer }\end{array}$ & Inception v3 & $\begin{array}{l}\text { Accuracy }-90.91 \% \\
\text { Sensitivity }-91.18 \% \\
\text { Specificity }-90.64 \%\end{array}$ \\
\hline 3 & Guimarães et al. [71] & 2019 & OGDE images & 200 & $\begin{array}{l}\text { Detection of gastric } \\
\text { precancerous condition }\end{array}$ & $\mathrm{CNN}$ & Accuracy $-93 \%$ \\
\hline 4 & Wang et al. [72] & 2019 & Gastroscopy images & 104864 & Screening of gastric cancer & CNN and SVM & Accuracy - $92.10 \%$ \\
\hline 5 & Gao et al. [73] & 2019 & tomography images & 1371 & $\begin{array}{l}\text { Detection of metastatic } \\
\text { lymph nodes for gastric } \\
\text { cancer classification }\end{array}$ & Faster region based CNN & Accuracy $95.45 \%$ \\
\hline 6 & Leon et al. [74] & 2019 & Histopathological images & 40 & Detection of gastric cancer & $\mathrm{CNN}$ & Accuracy $-89.72 \%$ \\
\hline 7 & Cho et al. [75] & 2019 & Endoscopic images & 5017 & $\begin{array}{l}\text { Detection of gastric } \\
\text { neoplasms }\end{array}$ & Inception Resnet v2 model & Accuracy - $84.6 \%$ \\
\hline 8 & Wu et al. [63] & 2018 & OGDE images & 24549 & $\begin{array}{l}\text { Early detection of gastric } \\
\text { cancer }\end{array}$ & Deep CNN & $\begin{array}{l}\text { Accuracy - } 92.5 \% \\
\text { Sensitivity }-94 \% \\
\text { Specificity }-91 \%\end{array}$ \\
\hline 9 & Sakai et al. [76] & 2018 & Endoscopic images & 926 & $\begin{array}{l}\text { Automatic detection of } \\
\text { gastric cancer }\end{array}$ & Transferring CNN & Accuracy $-82.8 \%$ \\
\hline 10 & Zhu et al. [67] & 2018 & Endoscopic images & 790 & $\begin{array}{l}\text { Prediction of invasion depth } \\
\text { for endoscopic resection }\end{array}$ & $\begin{array}{l}\text { CNN - computer aided } \\
\text { detection system }\end{array}$ & $\begin{array}{l}\text { Accuracy - } 89.66 \% \\
\text { Sensitivity }-76.47 \% \\
\text { Specificity }-95.56 \%\end{array}$ \\
\hline
\end{tabular}

OGDE images - Oesophagogastroduodenoscopic images, SVM - Support Vector Machine, CNN - Convolution Neural Network. 
in detecting tumors. This improvement in combination with $\mathrm{AI}$ analysis prevents the revisiting of patients with decreased false-positive rates $[4,18]$. Moreover, advancements in digital pathology will be more quantitative than radiology [19]. With more advancements in AI, we expect designing of FDA approved standard protocols for cancer diagnosis, classification, and management.

\section{Methodology}

In order to manifest the potential of artificial intelligence in cancer imaging, the following case studies were described in this paper:

- Application of artificial intelligence techniques detection and classification of

- lung cancer

- Breast cancer

- Gastric cancer

Furthermore, to show the advancements of AI in the field of cancer, the following search terms were used in the advanced search tool [20]:

- Application of artificial intelligence in lung cancer imaging technique

- Application of artificial intelligence in breast cancer imaging technique

- Application of artificial intelligence in gastric cancer imaging technique

The evolution of artificial intelligence in cancer imaging was carried out for a period of five years (January 2015 to May 2020). In order to understand the significance of AI in cancer, the graphical analysis of the evolution of $\mathrm{AI}$ in cancer imaging in the corresponding cancer type is illustrated in Fig. 3. In particular, the recent development of $\mathrm{AI}$ in cancer imaging techniques after 2017 is briefly discussed in this study. Selective research works having valuable contributions in the lung, breast and gastric cancer diagnosis and classification is tabulated in Table 1, 2 and 3 respectively.

\section{Results and Discussion}

Recent statistical analysis had reported a drastically increased death rate in lung cancer, breast cancer and gastric cancer worldwide [21]. Hence, early detection and classification with high accuracy had become essential. In turn, the contribution of researchers towards detection and classification had been increased. Fig. 3 shows the significance of $\mathrm{AI}$ in cancer imaging by researchers.

\subsection{Current trends in lung cancer diagnosis and classification using artificial intelligence}

Lung adenocarcinoma (LADC) being the foremost cause of death in humans among all other cancers. The morphological features of LADC are heterogeneous in nature, thus provides variation during diagnosis. Depending on the tumor size and lymph location, lung cancer is classified into four stages: stage 1 to stage 4 . The survival of victim depends on stages of the cancer which enhances the survival rate of the victim [22]. Hence early diagnosis plays a crucial role in lung cancer.

The preliminary diagnosis of lung cancer depends on the detection of pulmonary nodules. The computed tomography of both benign and malignant tumors contains pulmonary nodules. Hence differentiating those into benign and malignant using visual assessments by the radiologists had become challenging [23]. Moreover, nodule size is the most reliable prognosticator of malignancy in lung cancer. Hence nodule detection had become crucial in early diagnosis.

Till now, the tissue imaging process is playing an essential role in lung cancer prognosis. This strategy depends on morphological features such as tumor size, shape, and invasion of the tumor cells. It is also to be noted that these techniques lack a systematic approach of correlating the features to disease diagnosis [24]. Additionally, other diagnosing systems such as magnetic resonance imaging (MRI), sputum cytology, and chest radiography resulted in poor patient survival due to its lower classification accuracy and higher classification error rate. Hence the application of AI emerged as an effective tool in lung cancer diagnosis and classification.

Recently artificial intelligence is playing a prominent role in tumor detection, segmentation and classification as well as nodule detection in lung cancer. Moreover, tumor classification and metastasis detection from $H \& E$ images have been facilitated using deep learning strategies. For instance, Wang et al. and his co-workers developed a shape-based diagnostic model based on features such as age, gender, smoking status, and disease stage. The ultimate aim of the work to characterize the shape of the tumor, which is closely associated with disease prognosis. A convolution neural network was developed using 539 pathology images of lung adenocarcinoma patients obtained from NLST and TCGA repository. The generated model was validated using a subset of 389 images and the tumors were detected. The shape features of the tumor were extracted and analyzed. In addition, the risk score was used to group the people into low risk 
and high risk respectively. Moreover Kaplan - Meier method was to predict the survival rate of the patients. Around $89.8 \%$ accuracy was obtained for the prediction of tumor [25]. This study significantly proves the application of $\mathrm{AI}$ in lung cancer diagnosis.

Recently, a combined technique of neural network was proposed by Senthil et al. and his research scholar to enhance the classification accuracy with minimal error rate. They combined particle swarm optimization (PSO) algorithm to the neural network classifier. PSO is a highly cost-effective computational strategy with high speed. The accuracy, specificity and sensitivity of the proposed PSOneural network (PSO-NN) was compared with the standard neural networks such as k-means neural network (KNN), Bayes network (BN), neural network (NN) and support vector machine (SVM). Accuracy of PSO-NN was about $97.8 \%$, whereas the accuracy of KNN, BN, NN and SVM was found to be $68.9 \%, 74.6 \%, 85.4 \%$ and $91.5 \%$ respectively [26]. The proposed network suggests that AI can be effectively implemented for lung cancer diagnosis. Moreover it assists doctors in diagnosing and medicate patients at the earliest.

Besides the classification of lung cancer patients, the identification of mutations plays an important role in targeted therapy. The deep learning convolutional neural network model built using 1634 histopathology images from TCGA repository assisted the experts in treating patients through targeted therapy. In this study, Nicolas et al. used v3 convolutional neural network to classify disease and to identify the mutations from the images. Using the proposed model, he achieved around $97 \%$ of specificity and sensitivity for classifying the samples and $86 \%$ accuracy for identifying the mutations from the samples. His findings suggested the experts to cancer types and mutation with high accuracy and less expensive strategy [27]. Thus this proposed work can provide a promising treatment for lung cancer patients. Additionally, some research works contributed to lung cancer diagnosis, and classification were tabulated in Table 1 .

\subsection{Artificial Intelligence in diagnosis and classification of breast cancer}

Breast cancer holds the second position amongst overall cancer death worldwide. It occurs due to uncontrolled and abnormal growth of tissues resulting in lump formation in the breast. Thus it consequently leads to tumor growth which can be treated successfully at their early stages [35]. Recently, the most prominent tool "mammography" has been developed for early diagnosis of breast cancer. Regardless mammography had reduced mortality rate by $30 \%$, it contained certain limitations, including high false-positive rates, unnecessary biopsies, overdiagnosis, and treatment [36].

In addition, analyzing of a mammogram differs based on the experience and is always found to be biased due to dense breast tissues [37]. Subsequently, this leads to interval cancers that are detected prior to biennial screening [38].

Similarly, the presence of masses and microcalcification (calcium deposition) interferes with the quality of mammograms. Moreover, the detection of masses is more challenging than microcalcification due to its variation in size and shape, which in turn produces poor contrast images during mammography. This made the radiologists challenging to classify them as benign and malignant [39]. Thus automated image detection and classification is playing a vital role nowadays.

ANN is one of the most widely used tools for interpreting and decision making of mammography as well as biopsy screenings. The two major applications of AI in breast cancer are feature analysis from the images and to implement classifiers over the desired target [40].

In general, morphology of cells and its components are regulated by biological mechanisms such as differentiation, growth, and development. Earlier pathologists performed tedious visual approaches for tumor grading and morphological assessment of samples. This created larger variations amongst the senior pathologists [41]. Hence many strategies were developed for automated image analysis which includes CAD till AI. Recently, AI based strategies were found to be outperforming in pathology image analysis [42]. For instance, Rakhlin and his research group drawn attention for image feature extraction and their classification. Deep convolution neural network was implemented for extraction of image features, and gradient boosted trees classified them into 2 class as well as 4 class classifications respectively. Their study reported about $93.8 \%$ accuracy and $97.3 \%$ AUC with respect to 2-class classification tasks, whereas the 4-class classification reported only about $87.2 \%$ accuracy. Their strategy implemented unsupervised learning for feature extraction and supervised learning for classification. This study shows a significant result for feature extraction and classification during pathology image analysis [43].

In addition, the presence of highly dense breasts acts as a risk marker during imaging process. It describes the measure of intensity of fibro-glandular tissue in the breast. Moreover presence of dense breast masks cancer and thus reduces the sensitivity 
during imaging. Besides, the manual classification of dense breast images into four qualitative categories based on Breast Imaging and Reporting Data Systems (BI-RADS) by the radiologists was found to be more difficult [44]. Several studies using deep learning have been investigated in mammography imaging processes. Mohamed et al. and his group investigated a novel Convolution Neural Network (CNN) for classifying mammograms into scattered density and heterogeneously dense samples. The classification performance of $\mathrm{CNN}$ was also tested using the refined mammograms during their investigation. The AUC of the CNN model was $94.2 \%$. They observed that increasing the number of mammogram images increased their accuracy from $94.2 \%$ to $98.8 \%$. Hence their study demonstrated the classification accuracies between the two groups, which subsequently enhances clinical assessment of breast densities [45].

Despite the advancements in breast imaging techniques, interpreting the patterns have become more challenging. Besides, it required specialization and experience. However, the performance by the radiologists has been depleted due to the high incidence rate of breast cancer. At the same time, increased false-positive and recall rates had criticized the application of mammography for imaging and screening. Regardless of their drawbacks, mammograms have the property of being singleslice projection images which can be trained easily using ANN [46]. Becker et al. illustrated a combinatorial approach of deep learning with $\mathrm{CNN}(\mathrm{d}-$ $\mathrm{CNN}$ ) for diagnosing the images with higher accuracy and shorter period of time. They compared the accuracy of $\mathrm{d}-\mathrm{CNN}$ with the experienced radiologists. The study reported about $82 \%$ AUC for the model and about $79 \%$ AUC for radiologists respectively. The suggested model for analyzing general mammograms of breast cancer was higher than the radiologists. In a similar manner, the study suggested that improving the models will assist radiologists and makes clinical assessments easier [47]. Further, the contributions of other researchers are tabulated in Table 2.

\subsection{Recently proposed artificial intelligence strategies in gastric cancer diagnosis and classification}

Gastric cancer positions third as a fatal disease and fifth for its high incidence rate in the world [58]. The patients with early gastric cancer rarely bear the symptoms, but later on, the symptoms progress gradually. Moreover, the symptoms are much similar to gastric ulcers, thus it makes it difficult for the patients to differentiate them. This pre-requisites the need for early diagnosis of gastric cancer. The advancement in endoscopy assists in early diagnosis and substantially reduces the mortality rate of gastric cancer. Further, early detection helps us to remove the lesions using endoscopic resection, which significantly improves the patient's health quality [59]. Therefore it is essential to attain early diagnosis with high accuracy for identifying lesions to prevent and treat gastric cancer.

Magnifying endoscopy with narrow band imaging (M-NBI) is used to inspect glandular epithelium by observing microsurface structure and microvascular architecture. It has better accuracy than light endoscopy to distinguish non-cancerous and gastric cancer lesions [60]. Several investigations had reported that the sensitivity and specificity of identifying gastric cancer lesions using M-NBI was 85.7$97.3 \%$ and $84.4-96.8 \%$ respectively [61]. However, the differentiation ability between cancerous and non-cancerous lesions using M-NBI by non-experts was disappointing. To prevail over the limitations above, AI was implemented to enhance the accuracy of medical diagnosis.

Endoscopy is a widely used tool for diagnosing early stage of gastric cancer, among which $7.2 \%$ of patients were misdiagnosed. The meager changes in mucosa are often not detected during endoscopic analysis. This requires trained endoscopists with well-armed knowledge [62]. Hence Deep Convolution Neural Network (DCNN) gained attention for predicting and analyzing endoscopic images. $\mathrm{Wu}$ et al. detected early gastric cancer lesions with $92.5 \%$ accuracy and $94 \%$ specificity using DCNN. The diagnosing accuracy and stability were higher than the trained endoscopists. The time taken by DCNN model for diagnosing the lesions was comparatively lower than the endoscopists. Additionally, his study supported the generation of grid model over the stomach which covers the suspicious lesion regions too [63].

DL technology was initially implemented by Hirasawa et al. for the diagnosis of early gastric cancer in the year 2018. Researchers used images from conventional endoscope rather than from FICE and magnifying NBI. Single-shot multi-box pattern was used for the construction of CNN model. About 13,000 images were used as training set and 2296 images as test set for validating the performance of the model. Around 77 images in the test set were found with the lesions. The model categorized the test set in 47 seconds by finding 71 lesions containing images accurately with $92 \%$ overall sensitivity of the system. The CNN model detected and processed a stockpile of endoscopic images [64]. 
Endoscopic submucosal dissection is another approach for treating gastric cancer with minimal invasive depth. This technique is highly preferable by the patients since it requires shorter stay at hospitals [65]. However, endoscopic submucosal dissection are performed to the patients with invasive depth till submucosal layer of stomach based on the guidelines. In addition, the invasion depth can also be determined using endoscopic interventions for early gastric cancer by spraying indigo carmine dye. Moreover, the conventional endoscopy resulted in $69 \%$ to $79 \%$ accuracy [66]. This shows that there is no reliable tool available for measuring the invasive depth in gastric cancers. Recently CAD has been implemented for differential diagnosis of diseases. In 2018, Zhu et al. determined the invasive extent using CNN - CAD through transfer learning. About $89.16 \%$ accuracy was achieved in measuring the invasive depth with $76.47 \%$ sensitivity and $95.56 \%$ specificity. The developed CNN - CAD model differentiated earlier gastric cancer from submucosal invasion. This study also reduced misconception of invasion depth, which reduces trivial gastrectomy procedures [67]. Other contributions in gastric cancer imaging are consolidated in Table 3.

Another investigation by Kanesaka et al. improved the strategy by developing software to identify and delineate the boundaries between cancerous and non-cancerous regions. Support vector machines were implemented in this study to analyze the gray-level matrix features of narrowband images. One hundred twenty-six images were used as a training set, and 81 images were used as a test set for constructing the model. The sensitivity and specificity of the model for cancer from noncancerous imaged were validated and found to be $97 \%$ and $95 \%$, respectively. Similarly, the sensitivity and specificity of area concordance were $66 \%$ and $81 \%$, respectively [68].

\section{Conclusion}

Artificial intelligence has emerged as a powerful tool in cancer prognosis and management. Advancements in AI can be effectively implemented in personalized treatment and monitoring patient's health leading to a higher quality of care. Other areas in cancer such as follow up of patient's health, biochemical tests require the assistance of AI to help clinicians. Although the application of AI is beneficial, correct steps must be taken for framing the workflow in concern with the medical context to prevent undesired side effects in patients. Over time, this strategy may become a standard approach for human incidental disease diagnosis, evaluation, and reporting of data.

For bioinformaticians, image computing and machine learning assists in discovering prominent features for diagnosis and treatment, overcoming the aforementioned opportunities and challenges. It also assists researchers in developing new algorithms for disease characterization using radiology, molecular, and histology data. In this study, the limitations in the diagnosis and management of cancer diagnosis and classification was addressed. Also, the application of $\mathrm{AI}$ in tumor detection and classification using diagnostic images was discussed. A constant increase in the number and complexity of cancer images has reduced the time for evaluating the images by the radiologists. Due to the massive volume of images, prediction of tumors at the earliest has become one of the significant areas to be influenced by artificial intelligence. Moreover, progress in AI research will provide more solutions to the radiologists for predicting cancers. In the mere future, artificial intelligence will transcend as a prominent tool in clinical research.

\section{Conflict of interest}

The authors declare that they have no known competing financial interests or grants that could have appeared to influence the work reported in this paper.

\section{Acknowledgements}

The authors grately acknowledge Vellore Institute of Technology for providing the facilities and support to carry out this work.

\section{References}

[1] Bray F, Ferlay I, Soerjomataram I, Siegel RL, Torre LA, Jemal A. Global cancer statistics 2018: GLOBOCAN estimates of incidence and mortality worldwide for 36 cancers in 185 countries. Cancer J Clin 2018;68(6):394-424.

[2] Singh H, Sethi S, Raber M, Petersen LA. Errors in cancer diagnosis: current understanding and future directions. Int J Clin Oncol 2007;25(31):5009-18.

[3] Bi WL, Hosny A, Schabath MB, Giger ML, Birkbak NJ, Mehrtash A, et al. Artificial intelligence in cancer imaging: clinical challenges and applications. CA: Cancer J Clin 2019; 69(2):127-57.

[4] Hosny A, Parmar C, Quackenbush J, Schwartz LH, Aerts HJWL. Artificial intelligence in radiology. Nat Rev Canc 2018;18:500-10.

[5] Rolnick D, Veit A, Belongie S, Shavit N. Deep learning is robust to massive label noise. 2017. arXiv preprint arXiv: 1705.10694.

[6] Ziaei A, Mehri R, Salehi M. Application of artificial neural networks in cancer classification and diagnosis prediction of a subtype of lymphoma based on gene expression profile. Int J Res Med Sci 2006;11(1):13-7. 
[7] Agrawal S, Agrawal J. Neural network techniques for cancer prediction: A survey. Procedia Comput Sci 2015;60:769-74.

[8] Coccia M. Deep learning technology for improving cancer care in society: New directions in cancer imaging driven by artificial intelligence. Technol Soc 2020;60:101198.

[9] Castellino RA. Computer aided detection (CAD): an overview. Canc Imag 2005;5(1):17.

[10] Liang M, Tang W, Xu DM, Jirapatnakul AC, Reeves AP, Henschke CI, et al. Low-dose CT screening for lung cancer: computer-aided detection of missed lung cancers. Radiology 2016;281(1):279-88.

[11] Warfield SK, Zou KH, Wells WM. Validation of image segmentation by estimating rater bias and variance. Phil Trans Roy Soc Math Phys Eng Sci 2008;366(1874):2361-75.

[12] Mirsadraee S, Oswal D, Alizadeh Y, Caulo A, van Beek EJ. The 7th lung cancer TNM classification and staging system: Review of the changes and implications. World J Radiol 2012; 4(4):128.

[13] Litjens G, Sánchez CI, Timofeeva N, Hermsen M, Nagtegaal I, Kovacs I, et al. Deep learning as a tool for increased accuracy and efficiency of histopathological diagnosis. Sci Rep 2016;6:26286.

[14] Jiang F, Jiang Y, Zhi H, Dong Y, Li H, Ma S, et al. Artificial intelligence in healthcare: past, present and future. Stroke Vasc Neurol 2017;2(4):230-43.

[15] Khan S, Islam N, Jan Z, Din IU, Rodrigues JJ. A novel deep learning based framework for the detection and classification of breast cancer using transfer learning. Pattern Recognit Lett 2019;125:1-6.

[16] Kim DW, Lee S, Kwon S, Nam W, Cha IH, Kim HJ. Deep learning-based survival prediction of oral cancer patients. Sci Rep 2019;9.

[17] Gulshan V, Peng L, Coram M, et al. Development and validation of a deep learning algorithm for detection of diabetic retinopathy in retinal fundus photographs. JAMA 2016;316: 2402-10.

[18] Jochelson MS. Use of contrast-enhanced mammography in breast cancer screening. In: Contrast-Enhanced Mammography. Cham: Springer; 2019. p. 115-32.

[19] Scherer K, Willer K, Gromann L, Birnbacher L, Braig E, Grandl S, et al. Toward clinically compatible phase-contrast mammography. PloS one 2015;10(6).

[20] Coccia M. Artificial intelligence technology in cancer imaging: clinical challenges for detection of lung and breast cancer. J Soc Adm Sci 2019;6(2):82-98.

[21] Liu Y, Wang H, Gu Y, Lv X. Image classification toward lung cancer recognition by learning deep quality model. J Vis Commun Image 2019;63:102570.

[22] Soniya G, Priya CS, Divya V, Mohana J. Detection of lung cancer and its stages using random tree classifier. Int J Pure Appl Math 2018;120(6):10565-76.

[23] Carter BW, Godoy MC, Erasmus JJ. Predicting malignant nodules from screening CTs. J Thorac Oncol 2016;11(12): 2045-7.

[24] Matsuda T, Machii R. Morphological distribution of lung cancer from Cancer Incidence in Five Continents Vol. X. JPN J Clin Oncol 2015;45(4):404.

[25] Wang S, Chen A, Yang L, Cai L, Xie Y, Fujimoto J, et al. Comprehensive analysis of lung cancer pathology images to discover tumor shape and boundary features that predict survival outcome. Sci Rep 2018;8(1):1-9.

[26] Senthil S, Ayshwarya B. Lung cancer prediction using feed forward back propagation neural networks with optimal features. Int J Appl Eng 2018;13(1):318-25.

[27] Coudray N, Ocampo PS, Sakellaropoulos T, Narula N, Snuderl M, Fenyö D, et al. Classification and mutation prediction from non-small cell lung cancer histopathology images using deep learning. Nat Med 2018;24(10):1559-67.

[28] ALzubi JA, Bharathikannan B, Tanwar S, Manikandan R, Khanna A, Thaventhiran C. Boosted neural network ensemble classification for lung cancer disease diagnosis. Appl Soft Comput 2019;80:579-91.
[29] Pandiangan T, Bali I, Silalahi AR. Early lung cancer detection using artificial neural network. At Indones 2019;45(1):9-15.

[30] Nasser IM, Abu-Naser SS. Lung cancer detection using artificial neural network. Int J Eng Inf Syst 2019;3(3):17-23.

[31] Roy K, Chaudhury SS, Burman M, Ganguly A, Dutta C, Banik S, et al. A Comparative study of Lung Cancer detection using supervised neural network. Optronix 2019:1-5.

[32] Bhalerao RY, Jani HP, Gaitonde RK, Raut V. A novel approach for detection of lung cancer using digital image processing and convolution neural networks. In: 5th International Conference on Advanced Computing \& Communication Systems; 2019. p. 577-83.

[33] Perumal S, Velmurugan T. Lung cancer detection and classification on CT scan images using enhanced artificial bee colony optimization. Int J Eng Technol 2018;7:74-9 (2.26).

[34] Li X, Hu B, Li H, You B. Application of artificial intelligence in the diagnosis of multiple primary lung cancer. Thorac Cancer 2019;10(11):2168-74.

[35] Balakumaran T, Vennila IL, Shankar CG. Detection of microcalcification in mammograms using wavelet transform and fuzzy shell clustering. Int J Comput Eng Inf Technol 2010;7(1):121-5.

[36] Le EP, Wang Y, Huang Y, Hickman S, Gilbert FJ. Artificial intelligence in breast imaging. Clin Radiol 2019;74(5):357-66.

[37] Elmore JG, Jackson SL, Abraham L, Miglioretti DL, Carney PA, Geller BM, et al. Variability in interpretive performance at screening mammography and radiologists characteristics associated with accuracy. Radiology 2009; 253(3):641-51.

[38] Houssami N, Hunter K. The epidemiology, radiology and biological characteristics of interval breast cancers in population mammography screening. NPJ Breast Canc 2017;3(1): $1-3$.

[39] Mehdy MM, Ng PY, Shair EF, Saleh NI, Gomes C. Artificial neural networks in image processing for early detection of breast cancer. Comput Math Method; 2017.

[40] Ayer T, Chen Q, Burnside ES. Artificial neural networks in mammography interpretation and diagnostic decision making. Comput Math Method; 2013.

[41] Bejnordi BE, Veta M, Van Diest PJ, Van Ginneken B, Karssemeijer N, Litjens G, et al. Diagnostic assessment of deep learning algorithms for detection of lymph node metastases in women with breast cancer. Jama 2017;318(22): 2199-210.

[42] Robertson S, Azizpour H, Smith K, Hartman J. Digital image analysis in breast pathology - from image processing techniques to artificial intelligence. Transl Res 2018;194: 19-35.

[43] Rakhlin A, Shvets A, Iglovikov V, Kalinin AA. Deep convolutional neural networks for breast cancer histology image analysis. Int Conf Image Anal Recogn 2018:737-44.

[44] Mohammadpoor M, Shoeibi A, Shojaee H. A hierarchical classification method for breast tumor detection. Iran J Med Phys 2016;13(4):261-8.

[45] Mohamed AA, Berg WA, Peng H, Luo Y, Jankowitz RC, Wu S. A deep learning method for classifying mammographic breast density categories. Med Phys 2018;45(1): 314-21.

[46] Kuhl CK. The changing world of breast cancer: a radiologist's perspective. Invest Radiol 2015;50(9):615.

[47] Becker AS, Marcon M, Ghafoor S, Wurnig MC, Frauenfelder T, Boss A. Deep learning in mammography: diagnostic accuracy of a multipurpose image analysis software in the detection of breast cancer. Invest Radiol 2017; 52(7):434-40.

[48] Batra K, Sekhar S, Radha R. Breast cancer detection using CNN on mammogram images. Int Conf Comput Vision Bio Inspired Comput 2019:708-16.

[49] Ali AA, Mishra S, Dappuri B. Breast cancer classification using tetrolet transform based energy features and K-nearest neighbor classifier. In: Recent Trends and Advances in Artificial Intelligence and Internet of Things; 2020. p. 39-46. 
[50] Kim HE, Kim HH, Han BK, Kim KH, Han K, Nam H, et al. Changes in cancer detection and false-positive recall in mammography using artificial intelligence: a retrospective, multireader study. Lancet Glob Health 2020;2(3):138-48.

[51] Wadkar K, Pathak P, Wagh N. Breast cancer detection using ann network and performance analysis with SVM. J Comp Eng Technol 2019;10(3):75-86.

[52] Rodríguez-Ruiz A, Krupinski E, Mordang JJ, Schilling K, Heywang-Köbrunner SH, Sechopoulos I, et al. Detection of breast cancer with mammography: effect of an artificial intelligence support system. Radiology 2019;290(2):305-14.

[53] Alickovic E, Subasi A. Normalized neural networks for breast cancer classification. Int Conf Med Bio Eng 2019: 519-24.

[54] Rodriguez-Ruiz A, Lång K, Gubern-Merida A, Broeders M, Gennaro G, Clauser P, et al. Stand-alone artificial intelligence for breast cancer detection in mammography: comparison with 101 radiologists. J Natl Cancer Inst 2019;111(9): 916-22.

[55] Watanabe AT, Lim V, Vu HX, Chim R, Weise E, Liu J, et al. Improved cancer detection using artificial intelligence: a retrospective evaluation of missed cancers on mammography. J Digit Imag 2019;32(4):625-37.

[56] Wang Z, Li M, Wang H, Jiang H, Yao Y, Zhang H, et al, Breast cancer detection using extreme learning machine based on feature fusion with CNN deep features. IEEE Access 2019:105146-58.

[57] Huang MW, Chen CW, Lin WC, Ke SW, Tsai CF. SVM and SVM ensembles in breast cancer prediction. PloS one 2017; 12(1).

[58] Torre LA, Bray F, Siegel RL, et al. Global cancer statistics, 2012. CA Cancer J Clin 2015;65:87-108.

[59] Fujishiro M, Yoshida S, Matsuda R, Narita A, Yamashita H, Seto Y. Updated evidence on endoscopic resection of early gastric cancer from Japan. Gastr Canc 2017;20:39-44.

[60] Muto M, Yao K, Kaise M, Kato M, Uedo N, Yagi K, et al. Magnifying endoscopy simple diagnostic algorithm for early gastric cancer (MESDA-G). Dig Endosc 2016;28(4):379-93.

[61] Maki S, Yao K, Nagahama T, Beppu T, Hisabe T, Takaki Y, et al. Gastr Cancer 2013;16(2):140-6.

[62] Torkamani A, Andersen KG, Steinhubl SR, et al. High-definition medicine. Cell 2017;170:828-43.

[63] Wu L, Zhou W, Wan X, Zhang J, Shen L, Hu S, et al. A deep neural network improves endoscopic detection of early gastric cancer without blind spots. Endosc 2019;51:522-31 (06).

[64] Hirasawa T, Aoyama K, Tanimoto T, et al. Application of artificial intelligence using a convolutional neural network for detecting gastric cancer in endoscopic images. Gastr Cancer 2018;21:653-60.
[65] Isomoto H, Shikuwa S, Yamaguchi N, et al. Endoscopic submucosal dissection for early gastric cancer: a large-scale feasibility study. Gut 2009;58:331-6.

[66] Choi J, Kim S, Im J, et al. Comparison of endoscopic ultrasonography and conventional endoscopy for prediction of depth of tumor invasion in early gastric cancer. Endosc 2010 42:705-13.

[67] Zhu Y, Wang QC, Xu MD, Zhang Z, Cheng J, Zhong YS, et al. Application of convolutional neural network in the diagnosis of the invasion depth of gastric cancer based on conventional endoscopy. Gastrointest Endosc 2019;89(4):806-15.

[68] Kanesaka T, Lee TC, Uedo N, et al. Computer-aided diagnosis for identifying and delineating early gastric cancers in magnifying narrow-band imaging. Gastrointest Endosc 2018; 87:1339-44.

[69] Aslam MA, Xue C, Wang K, Chen Y, Zhang A, Cai W, et al. SVM based classification and prediction system for gastric cancer using dominant features of saliva. Nano Biomed Eng 2020;12(1):1-3.

[70] Li L, Chen Y, Shen Z, Zhang X, Sang J, Ding Y, et al. Convolutional neural network for the diagnosis of early gastric cancer based on magnifying narrow band imaging. Gastr Canc 2020;23(1):126-32.

[71] Guimarães P, Keller A, Fehlmann T, Lammert F, Casper M. Deep-learning based detection of gastric precancerous conditions. Gut 2020;69(1):4-6.

[72] Wang L, Yang S, Zhou A, Huang R, Ding S, Wang H, et al. An intelligent gastric cancer screening method based on convolutional neural network and support vector machine. Int J Comput Appl 2019;16:1-6.

[73] Gao Y, Zhang ZD, Li S, Guo YT, Wu OY, Liu SH, et al. Deep neural network-assisted computed tomography diagnosis of metastatic lymph nodes from gastric cancer. Chin Med J 2019;132(23):2804

[74] Leon F, Gelvez M, Jaimes Z, Gelvez T, Arguello H. Supervised classification of histopathological images using convolutional neuronal networks for gastric cancer detection. In: XXII Symposium on Image, Signal Processing and Artificial Vision. 2019; 2019. p. 1-5.

[75] Cho BJ, Bang CS, Park SW, Yang YJ, Seo SI, Lim H, et al. Automated classification of gastric neoplasms in endoscopic images using a convolutional neural network. Endosc 2019; 51(12):1121-9.

[76] Sakai Y, Takemoto S, Hori K, Nishimura M, Ikematsu H, Yano $\mathrm{T}$, et al. Automatic detection of early gastric cancer in endoscopic images using a transferring convolutional neural network. In: 40th Annual International Conference of the IEEE Engineering in Medicine and Biology Society; 2018. p. 4138-41. 